\title{
A Novel Animal Model of Nonalcoholic Steatohepatitis (NASH): Hypoxemia Enhances the Development of NASH
}

\author{
Fusako Takayama ${ }^{1, *}$, Toru Egashira ${ }^{2}$, Hiromu Kawasaki ${ }^{1}$, Mitsumasa Mankura ${ }^{2}$, \\ Kazuo Nakamoto ${ }^{1,2}$, Shigeru Okada ${ }^{2}$, and Akitane Mori ${ }^{2}$ \\ ${ }^{1}$ Department of Clinical Pharmaceutical Science, Graduate School of Medicine, \\ Dentistry and Pharmaceutical Sciences, Okayama University, Okayama 700-8530, Japan \\ ${ }^{2}$ Department of Anti-Aging Food Sciences, Graduate School of Medicine, Dentistry and Pharmaceutical Sciences, \\ Okayama University, Okayama 700-8530, Japan
}

Received 23 March, 2009; Accepted 17 June, 2009

\begin{abstract}
Summary Recent reports described a high incidence of nonalcoholic steatohepatitis (NASH) in patients with obstructive sleep apnea. Accordingly, we hypothesized that recurrent and intermittent hypoxemia plays an important role in the pathogenesis of NASH. Our objective was construction of a practical and accurate experimental model to reproduce the key features of NASH in humans. Chemical hypoxemia through methemoglobinemia was induced by daily intraperitoneal injection of sodium nitrite $(40 \mathrm{mg} / \mathrm{kg})$ for 4 weeks in rats with fatty liver. The later was induced by 4-week feeding a choline-deficient high-fat diet (CDHF). Besides, the normal chow diets feeding groups were prepared with in the same manner except for CDHF feeding. The animal experiment was performed in four groups; Normal control, Hypoxemia, CDHF, and CDHF + hypoxemia. Nitrite was given for the later 4 weeks to each rat of Hypoxemia and CDHF + hypoxemia. CDHF + hypoxemia rats were confirmed to develop histological changes that resemble those of patients with NASH, together with biochemical liver dysfunction, while CDHF group was limited in mild steatosis, and Hypoxemia group liver was normal. Present study established a reproducible and useful NASH model resembling the main features of NASH in humans, and showed first that recurrent and intermittent hypoxemia aggravate fatty liver to steatohepatitis and liver fibrosis.
\end{abstract}

Key Words: obstructive sleep apnea, hypoxemia, oxidative stress, methemoglobinemia

\section{Introduction}

Nonalcoholic steatohepatitis (NASH) is a progressive liver disorder that occurs in patients without significant alcohol consumption, and, histologically, it resembles alcoholic liver disease with macrovesicular steatosis, spotty necrosis, inflammation and fibrosis $[1,2]$.

Animal models have greatly contributed to the understanding of NASH [3-5]. Such models include rats that are

\footnotetext{
*To whom correspondence should be addressed.

Tel \& Fax: +81-86-251-7973

E-mail: takayamf@cc.okayama-u.ac.jp
}

treated for up to one year [6] or rats that are fed cholineand methionine-free diet [7], a nutritional deficiency to which rodents are highly sensitive but which is not common in patients with NASH. However, until recently, the study of therapeutics for NASH has been difficult because of the lack of a suitable experimental model.

Patients with obstructive sleep apnea (OSA) suffer from potentially severe hypoxemia due to repetitive airway obstruction during sleep, and are at risk of development of fatty liver as a result of obesity [8]. There is also evidence that OSA per se could be a risk factor for liver injury; ischemic hepatitis during OSA has been reported [9, 10].

Here we describe a new and more clinically-relevant rat model of NASH. Rats were fed a choline-deficient 
fat-rich diet and made hypoxemic to induce within a relatively short period of time liver dysfunction that resembled NASH. We present the histological and biochemical changes induced by the above treatment.

\section{Materials and Methods}

\section{Animals}

Six-week-old male Wistar rats were purchased from Shimizu Animal. Co. (Kyoto, Japan) and maintained at the Okayama University animal facility in accordance with the Okayama University Guidelines on the Care and Use of Laboratory Animals. Rats were housed in individual cages in air-conditioned rooms with controlled 12-h light-dark cycle. The rats were allowed to acclimate to the animal facility and provided free access to diets and water ad libitum.

\section{Experimental design}

At 10 weeks of age, the animals were divided into two groups and fed either control normal diets or cholinedeficient high-fat (CDHF) diets (Oriental Yeast Co., Tokyo, Japan) for 4 weeks. At the end of this period, rats of the Normal control group continued to be fed normal chow (MF laboratory chow, Oriental Yeast Co., Tokyo, Japan). On the other hand, rats of the CDHF + hypoxemia group received CDHF diets for another 4 weeks and treated during this period with intraperitoneal injection of $40 \mathrm{mg} / \mathrm{kg}$ of sodium nitrite $\left(\mathrm{NaNO}_{2}\right)$ every day to induce methemoglobinemia [11]. Rats of the Hypoxemia group were fed standard diets for another 4 weeks without $\mathrm{NaNO}_{2}$.

\section{Collection of biological samples}

At the end of the 8-week experimental period, the rats were sacrificed under light ether anesthesia, blood was collected from the inferior vena cava for serum analysis, and livers were resected for histopathological and biochemical analyses. In addition, blood samples were also drawn under light ether anesthesia, every week during the experimental feeding period. Besides, after the single intraperitoneal administration of $40 \mathrm{mg} / \mathrm{kg} \mathrm{NaNO} 2$ to rats fed with 4 weeks CDHF diets, the arterial bloods were drawn with time from femoral arteries cannulated with silicon tubes, and were kept on ice until determining the partial pressure of oxygen and the methemoglobin level.

\section{Biochemical analysis}

Serum biochemical markers, AST, ALT, $\gamma$-GTP, triglycerides and ALP were determined spectrophotometrically by using standard kits (Wako Pure Chemical Industries, Osaka, Japan). The levels of hyaluronic acid were measured in serum samples and liver homogenates using ELISA kits (SEIKAGAKU Corporation, Tokyo, Japan). Arterial partial pressure of oxygen was determined within $15 \mathrm{~min}$ of blood being drawn, with a blood gas analyzer (IRMA SL 2000, Diametrics Medical, Tokyo). The methemoglobin level was determined spectrophotometrically [11]. Blood glucose was determined by the glucose-oxidase method using Glucocard (Aventis Pharma, Tokyo, Japan).

\section{Histological examination}

Liver tissues were collected then fixed overnight in $10 \%$ formaldehyde in phosphate-buffered saline, then dehydrated and embedded in paraffin. Liver tissue sections ( $4 \mu \mathrm{m}$ thick) were stained with hematoxylin and with Masson trichrome (MT) staining for histological examination.

\section{Statistical analysis}

Data are expressed as mean \pm SEM of five rats. Differences among groups were examined by one-way analysis of variance (ANOVA) followed by Dunnett's multiple comparison test. A $p$ value less than 0.05 was considered statistically significant.

\section{Results}

Arterial partial pressure of oxygen and methemoglobin formation in rats fed choline-deficient hyper-fat diets and with intraperitoneal single $\mathrm{NaNO}_{2}$ injection

After the intraperitoneal single $40 \mathrm{mg} / \mathrm{kg} \mathrm{NaNO} 2$ injection to the rats fed CDHF for 4 weeks, in an arterial blood, there was induced the methemoglobin formation at the level of approximately $4 \mathrm{~g} / \mathrm{dL}$ in within $15 \mathrm{~min}$ of injection, followed by a decrease to less than $1 \mathrm{~g} / \mathrm{dL}$ at $2 \mathrm{~h}$ later. The blood methemoglobin concentrations correlated inversely with arterial partial pressure of oxygen (Fig. 1).

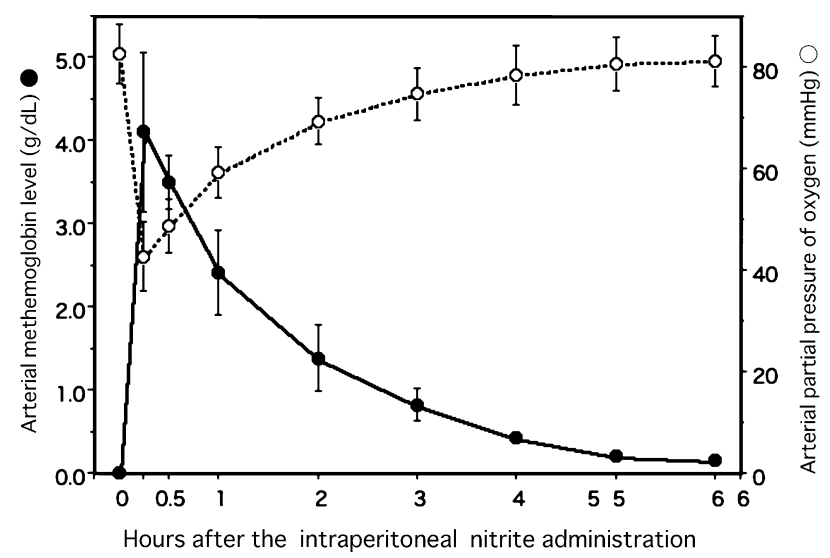

Fig. 1. Methemoglobin levels and arterial partial pressure of oxygen of rats after the single intraperitoneal administration of $40 \mathrm{mg} / \mathrm{kg} \mathrm{NaNO}$. The partial pressure of oxygen of blood samples were determined within $15 \mathrm{~min}$ after drawn from the cannulated femoral artery with PE-50 tube. Data are mean \pm SEM of four rats. 


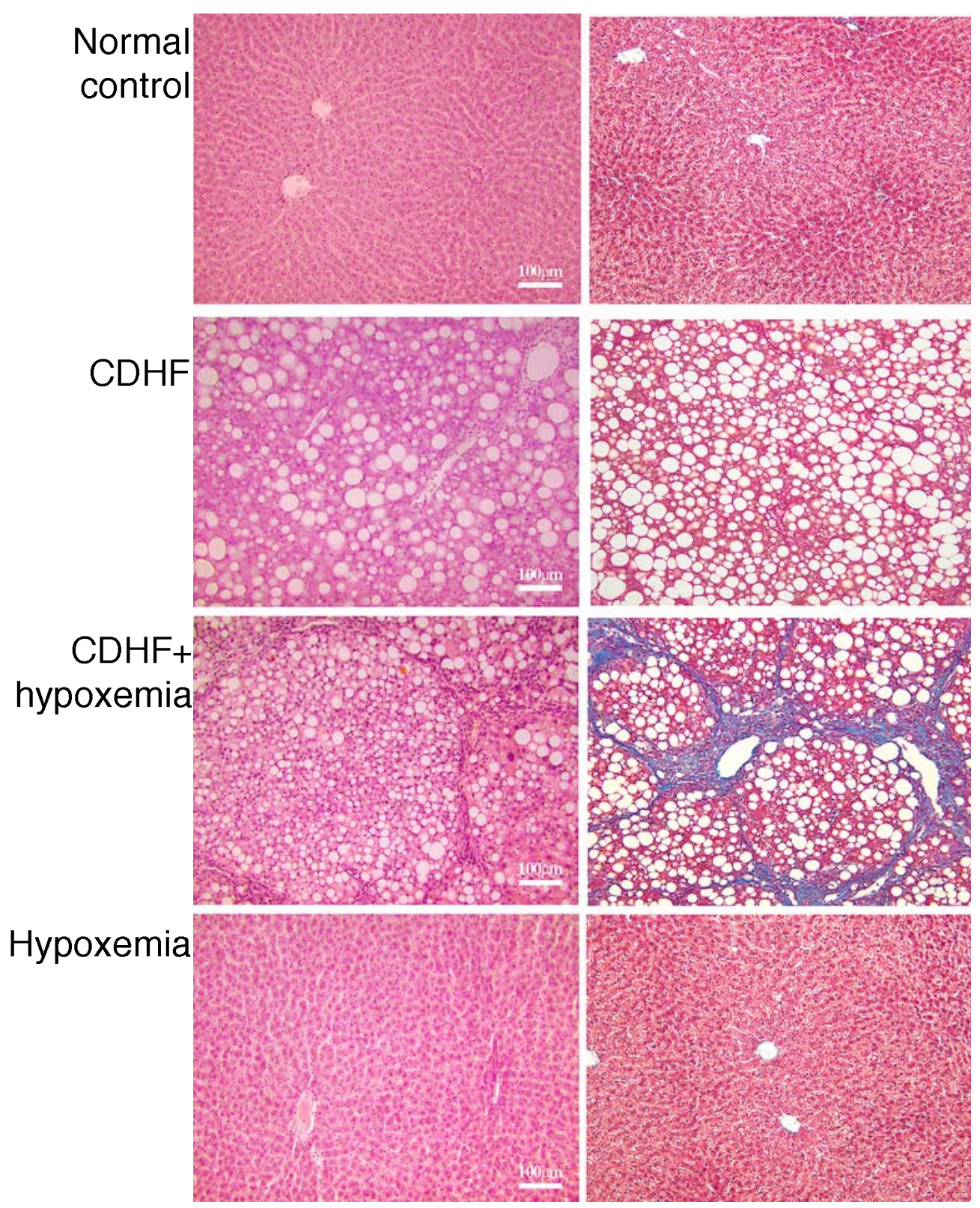

Fig. 2. Histology of liver tissues of the experimental groups. Liver sections were stained with hematoxylin (left) and Masson trichrome-stain (right). Note the extent of liver damage in the CDHF + hypoxemia rats.

Hepatic pathology in rats fed choline-deficient hyper-fat diets and exposed to hypoxia

Histological examination of livers of rats fed CDHF for 8 weeks showed marked steatohepatitis (Fig. 2, CDHF group). Fatty change was mostly a macrovesicular type, involving all zones of the lobe. Inflammatory infiltrates consisted of foci of acute and chronic inflammatory cells randomly distributed in the lobe. However, no liver fibrosis was present. On the other hand, severe liver fibrosis was observed in rats fed for 8 weeks on CDHF diets as well as $\mathrm{NaNO}_{2}$ treatment during the last 4 weeks (CDHF + hypoxemia group), (Fig. 2, right panel, stained with blue, $\mathrm{CDHF}+$ hypoxemia). Another control group; rats exposed to hypoxia alone and provided with normal diets showed no histopathological changes in liver tissue (Fig. 2, Hypoxemia). In the next series of experiments, the effects of the above histological changes on various biological markers of liver function were examined.

\section{Serum and liver biochemical parameters}

Serum triglyceride levels were comparable in control and hypoxemic rats (Table 1). On the other hand, at 8 weeks of treatment, hepatic triglyceride levels were significantly higher in CDHF and CDHF + hypoxemia rats than those fed normal chew (Normal control group) or treated with $\mathrm{NaNO}_{2}$ 
Table 1. Triglyceride levels in plasma and liver of rats fed control diets or choline-deficient hyper-fat diets with or without hypoxemic stress

\begin{tabular}{lcc}
\hline Group & $\begin{array}{c}\text { plasma } \\
(\mathrm{mg} / \mathrm{ml})\end{array}$ & $\begin{array}{c}\text { liver } \\
(\mathrm{mg} / \mathrm{g} \text { wet weight })\end{array}$ \\
\hline Normal control & $0.661 \pm 0.049$ & $2.439 \pm 0.249$ \\
CDHF & $0.418 \pm 0.055$ & $22.017 \pm 1.694^{* *}$ \\
CDHF + hypoxemia & $0.442 \pm 0.090$ & $17.787 \pm 2.216^{* *,+\dagger}$ \\
Hypoxemia & $0.650 \pm 0.051$ & $2.244 \pm 0.187$ \\
\hline
\end{tabular}

Each value represents the mean \pm SEM for five rats. Data were statistically analyzed by one-way analysis of variance (ANOVA) with Dunnet's multiple comparison test. ${ }^{* *} p<0.01$ vs Normal control group, ${ }^{\dagger} p<0.01$ vs Hypoxemia group.

alone (Hypoxemia group).

Body weights, blood glucose levels and survival rates at 8 weeks of treatment, are shown in Table 2. Body weights were significantly lower in CDHF and CDHF + hypoxemia rats than those fed normal chew (Normal control group) or treated with $\mathrm{NaNO}_{2}$ alone (Hypoxemia group). The blood glucose levels were not different between groups. Except for one of five CDHF + hypoxemia rats, all rats were alive at the end of the experimental period.

\section{Biochemical marker of liver fibrosis}

Serial measurements of serum hyaluronic acid showed no changes in its level at 4 weeks of control diets, CDHF alone, and hypoxemia alone. However, switching to the combination of CDHF and hypoxemia at week 4 gradually and significantly increased serum hyaluronic acid levels, which rose to 2.5 -fold those of rats of other groups at 8 weeks of treatment (Fig. 3).

\section{Changes in hepatobiliary enzymes}

Serum levels of both AST and ALT were significantly higher in the CDHF and CDHF + hypoxemia rats compared

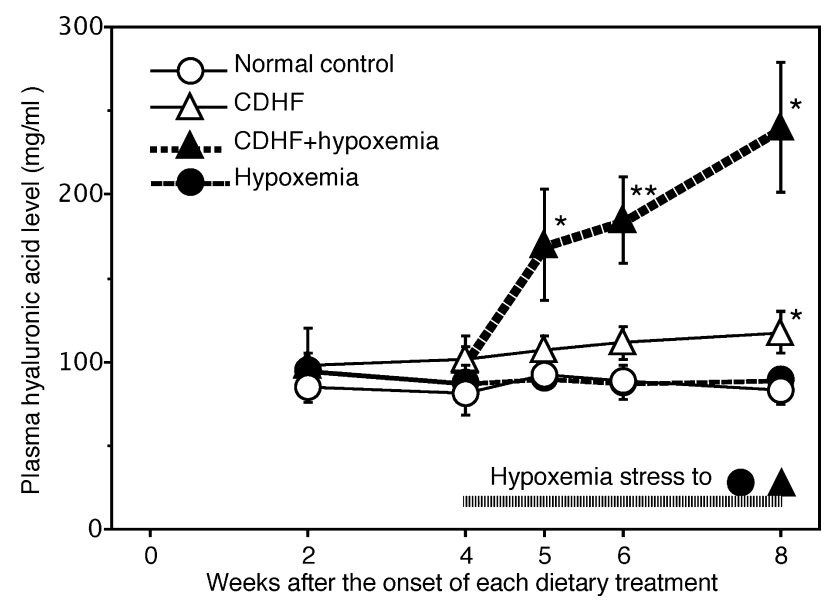

Fig. 3. Serial changes in serum hyaluronic acid levels in the four rat groups. Data are mean \pm SEM of five rats in each group. ${ }^{*} p<0.05,{ }^{*} p<0.01$ vs the value at 2 weeks for the corresponding group (by ANOVA followed by with Dunnett's multiple comparison test).

with the control and hypoxemia groups (Fig. 4A). In particular, AST and ALT levels in CDHF + hypoxemia rats were 5-fold and 2.7-fold higher, respectively, than the control group. Furthermore, serum ALP activity was significantly higher at 8 weeks in CDHF and CDHF + hypoxemia groups, compared with the other two groups (Fig. 4B). However, $\gamma$-GTP activity was only significantly increased in the CDHF + hypoxemia group, compared with the other groups (Fig. 4B).

\section{Discussion}

NASH can be associated with progressive hepatic fibrosis and is characterized by histopathological changes resembling alcohol-like liver damage in the absence of alcohol abuse. Several animal models of steatosis exist, but few models of steatohepatitis are available.

Table 2. Body weights, blood glucose levels and survival of rats fed control diets or choline deficient hyper-fat diets with or without hypoxemia stress

\begin{tabular}{llcc}
\hline Group & $\begin{array}{c}\text { body weght } \\
(\mathrm{g} / \text { body })\end{array}$ & $\begin{array}{c}\text { blood glucose } \\
(\mathrm{mg} / \mathrm{dL})\end{array}$ & $\begin{array}{c}\text { survival } \\
(\%)\end{array}$ \\
\hline Normal control & $307.3 \pm 6.7$ & $115.6 \pm 7.8$ & 100 \\
CDHF & $209.8 \pm 6.2^{\# \#}$ & $110.6 \pm 9.7$ & 100 \\
CDHF + hypoxemia & $217.8 \pm 13.6^{\# \#, \dagger \dagger}$ & $120.3 \pm 14.6$ & 80 \\
Hypoxemia & $313.2 \pm 6.7$ & $117.4 \pm 11.6$ & 100 \\
\hline
\end{tabular}

Normal control and Hypoxemia rats were fed on standard diets without and with hypoxemia stress, respectively. CDHF and CDHF + hypoxemia rats were fed on choline deficient hyperfat (CDHF) diets without and with hypoxemia stress, respectively. Each value represents the mean \pm SEM or the mean or five rats. Data were statistically analyzed by one-way analysis of variance (ANOVA) techniques with Dunnet's multiple comparison procedures. ${ }^{\#} p<0.01$ vs Normal control group, ${ }^{\dagger} p<0.01$ vs Hypoxemia group. 

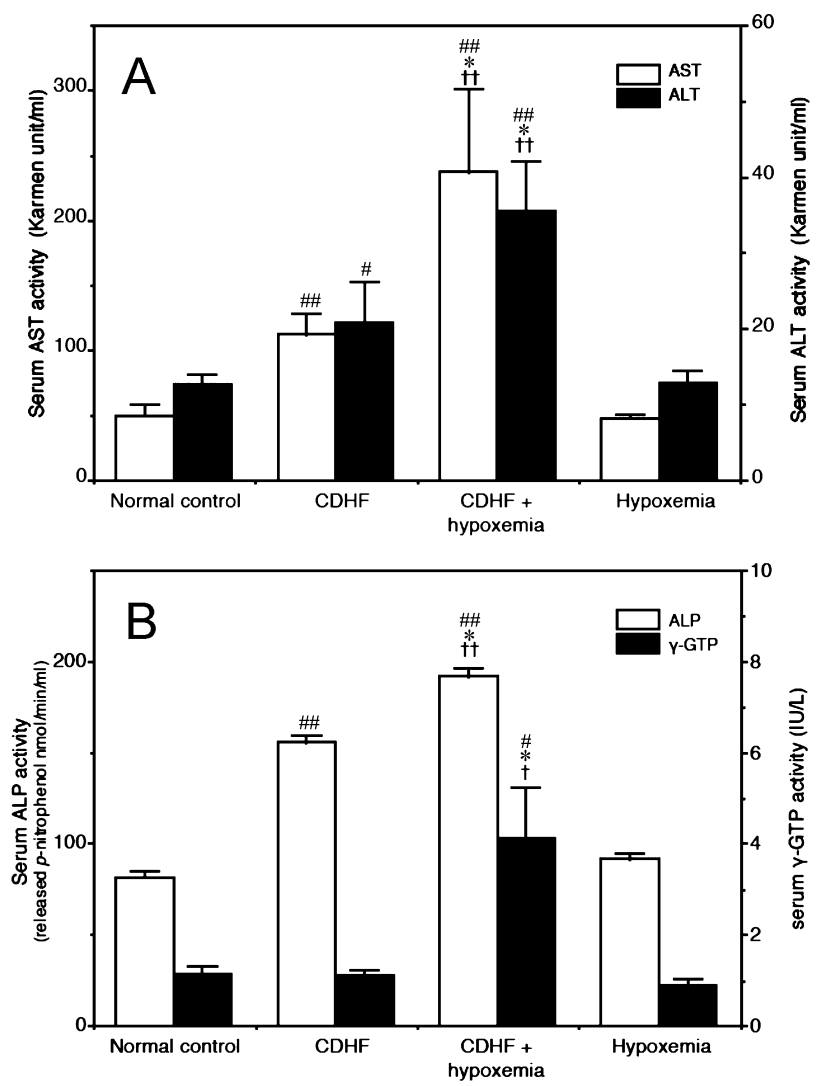

Fig. 4. Biochemical markers in serum for the hepatobiliary damages: AST and ALT levels (A), ALP and $\gamma$-GTP levels (B), in the four experimental groups measured at the end of the study period. Each value represents the mean \pm SEM of five rats. ${ }^{\#} p<0.05,{ }^{\#} p<0.01$ vs Normal control group, ${ }^{*} p<0.05,{ }^{* *} p<0.01$ vs CDHF group, ${ }^{\dagger} p<0.05$, ${ }^{\dagger} p<0.01$ vs Hypoxemia group (by ANOVA followed by with Dunnett's multiple comparison test).

Although the pathogenesis of NASH has not yet been fully elucidated, the "two-hit" theory is now accepted [12]. The first hit is the accumulation of fatty acids in the liver caused by several mechanisms (steatosis). Steatosis is relatively mild pathological process and is easily reversible. However, with secondary cellular stress (e.g., oxidative stress, endotoxin-mediated cytokine release), steatosis can progress to steatohepatitis, which is characterized by inflammation, necrosis, and fibrosis; i.e., a chronic liver disease $[13,14]$.

A methionine-choline deficiency model was used for $\mathrm{NASH}$, because the changes induced in the liver are reproducible, rapid and similar to those observed in NASH $[7,15,16]$. In our attempt to develop a NASH model, we were able to induce steatosis in rats fairly easily by feeding them at the age of 4 weeks CDHF diets continuously for 4 weeks. In the next step, we considered that oxidative stress produced by different methods could induce liver damage, similar to the reported hypoxemic-related liver injury in patients with OSA [8]. In fact, there is evidence to suggest that liver injury in OSA is the direct result of hypoxemia rather than ischemia $[9,10]$. Several studies argued that hypoxemia could play a role in the pathogenesis of NASH, $[17,18]$ suggesting that NASH represents tissue hypoxemia in fatty liver.

In this study, rats fed CDHF diets for 4 weeks followed by another 4 weeks of the same diets combined with $40 \mathrm{mg} / \mathrm{kg}$ $\mathrm{NaNO}_{2}$ demonstrated significant fatty deposits and leukocytic infiltration in the liver. Severe liver fibrosis was only observed in the CDHF + hypoxemia rats. Thus, by feeding rats a CDHF diet combined with $\mathrm{NaNO}_{2}$, we reproduced the typical hepatic lesions of NASH, namely, steatosis, inflammation, and early fibrosis, as indicated by a significant increase in triglyceride, hyaluronic acid, AST, ALT and $\gamma$-GTP.

We reported previously that hypoxemia was produced by administration of $\mathrm{NaNO}_{2}$ [11]. Indeed, nitrite is now being recognized as a critical 'hypoxic buffer', potentially contributing to the regulation of hypoxic vasodilatation and hypoxic mitochondrial respiration [19]. As well known, the methemoglobin cannot possess oxygen capacity, its increase in the blood, results in hypoxia. As shown Fig. 1, the arterial partial pressure of oxygen decreased to approximately $40 \mathrm{mmHg}$ within $15 \mathrm{~min}$ from injection, thereafter $1 \mathrm{~h}$ later, recovered to approximately $60 \mathrm{mmHg}$, the criteria level of the human respiratory failure. The $\mathrm{NaNO}_{2}$ injections were performed everyday, there rats were exposed to recurrent and intermittent hypoxia, in other words, repetitive cycles of hypoxia and reoxygenation, for 4 weeks. That is considered similar to recurrent ischemia-reperfusion (mild or moderate) led to oxidative stress in the liver, from the viewpoint of oxygen supply.

Although this study did not examine how the hypoxia induced liver fibrosis in the rats with simple fatty liver, and not in the controls, we suppose that resulted from the vulnerability of fatty liver to recurrent and intermittent hypoxia. Fatty liver may reduce tolerance to ischemiareperfusion injury. There are experimental studies that have indicated vulnerability of fatty hepatocytes to anoxic injury [20], and have shown the increased sensitivity to anoxia is caused by the fatty metamorphosis, when compared with normal liver parenchymal cells. The hypoxemia exposure to fatty liver leads to hepatocytes swelling via oxidative stress mechanisms, in there may proceed NF- $\mathrm{kB}$ activation inflammatory reaction and fibrosis.

Due to its high water-solubility, $\mathrm{NaNO}_{2}$ is mainly excreted via the kidney. We also confirmed histologically and biochemically that daily systemic injection of $\mathrm{NaNO}_{2}$ $(40 \mathrm{mg} / \mathrm{kg} /$ day) did not cause any renal injury or dysfunction (data not shown).

Our new rat model of NASH might be useful for the development of therapeutic agents against NASH. In addi- 
tion, this model (PCT/JP 2007/52477) suggests that patients with severe OSA could have subclinical steatosis and liver fibrosis.

\section{References}

[1] Ludwig, J., Viggiano, T.R., McGill, D.B., and Ott, B.J.: Nonalcoholic steatohepatitis. Mayo clinic experiences with a hitherto unnamed disease. Mayo Clin. Proc., 55, 434-438, 1980.

[2] Matteoni, C.A., Younossi, Z.M., Gramich, T., Boparai, N., Liu, Y.C., and McCullough, A.J.: Nonalcoholic fatty liver disease: a spectrum of clinical and pathological severity. Gastroenterology, 116, 1413-1419, 1999.

[3] Kirsch, R., Clarkson, V., Shephard, E.G., Marais, D.A., Jaffer, M.A., Woodburne, V.E., Kirsch, R.E., and Hall Pde, L.: Rodent nutritional model of non-alcoholic steatohepatitis: Species, strain and sex difference studies. J. Gastroenterol. Hepatol., 18, 1272-1282, 2003.

[4] Lieber, C.S., Leo, M.A., Mak, K.M., Xu, Y., Cao, Q., Ren, C., Ponomarenko, A., and DeCarli, L.M.: Model of nonalcoholic steatohepatitis. Am. J Clin. Nutr., 79, 502-509, 2004.

[5] Feldstein, A.E., Canbay, A., Guicciardi, M.E., Higuchi, H., Bronk, S.F., and Gores, G.J.: Diet associated hepatic steatosis sensitizes to Fas mediated liver injury in mice. J. Hepatol., 39, 978-983, 2003.

[6] Raucy, J.L., Lasker, J.M., Kramer, J.C., Salazar, D.E., Lieber, C.S., and Corcoran, G.B.: Induction of P450IIE1 in the obese rat. Mol. Pharmacol., 39, 275-280, 1991.

[7] Larter, C.Z., Yeh, M.M., Williams, J., Bell-Anderson, K.S., and Farrell, G.C.: MCD-induced steatohepatitis is associated with hepatic adiponectin resistance and adipogenic transformation of hepatocytes. J. Hepatol., 49, 407-416, 2008.

[8] Tanne, F., Gagnadoux, F., Chazouilleres, O., Fleury, B., Wendum, D., Lasnier, E., Lebeau, B., Poupon, R., and Serfaty, L.: Chronic liver injury during obstructive sleep apnea. Hepatology, 41, 1290-1296, 2005.

[9] Henrion, J., Colin, L., Schapira, M., and Heller, F.R.: Hypoxic hepatitis caused by severe hypoxemia from obstructive sleep apnea. J. Clin. Gastroenterol., 24, 245-249, 1997.

[10] Mathurin, P., Durand, F., Ganne, N., Mollo, J.L., Lebrec, D.,
Degott, C., Erlinger, S., Benhamou, J., and Bernuau, J.: Ischemic hepatitis due to obstructive sleep apnea. Gastroenterology, 109, 1682-1684, 1995.

[11] Egashira, T., Kaneko, O., Sawada, J., Kim, G., Sadaoka, K., and Sho, S.: Studies on monoamine oxidase (report 23) Effect of $\mathrm{NaNO}_{2}$ and $\mathrm{NH}_{2} \mathrm{OH}$ on monoamine oxidase in rat brain and liver. Folia Pharmacol. Jpn. (in Japanese with English abstract), 69, 969-976, 1973.

[12] Day, C.D. and James, O.F.W.: Steatohepatitis: a tale of two "hits"?. Gastroenterology, 114, 842-845, 1998.

[13] Tokushige, K., Takakura, M., Tsuchiya-Matsushita, N., Taniai, M., Hashimoto, E., and Shiratori, K.: Inuence of TNF gene polymorphisms in Japanese patients with NASH and simple steatosis. J. Hepatol., 46, 1104-1110, 2007.

[14] Burt, A.D., Mutton, A., and Day, C.P.: Diagnosis and interpretation of steatosis and steatohepatitis. Semin. Diagn. Pathol., 15, 246-258, 1998.

[15] Zhang, B., Weltman, M., and Farrell, G.C.: Does steatohepatitis impair liver regeneration? A study in a dietary model of non-alcoholic steatohepatitis in rats. J. Gastroenterol. Hepatol., 14, 133-137, 1999.

[16] Kashireddy, P. and Rao, S.M.: Sex differences in cholinedeficient diet induced steatohepatitis in mice. Exp. Biol. Med., 229, 158-162, 2004.

[17] Chalasani, N., Gorski, J.C., Asghar, M.S., Asghar, A., Foresman, B., Hall, S.D., and Crabb, D.W.: Hepatic cytochrome P450 2E1 activity in nondiabetic patients with nonalcoholic steatohepatitis. Hepatology, 37, 544-550, 2003.

[18] Barceló, A., Miralles, C., Barbé, F., Vila, M., Pons, S., and Agustí, A.G.: Abnormal lipid peroxidation in patients with sleep apnoea. Eur. Respir. J., 16, 644-647, 2000.

[19] Gladwin, M.T., Schechter, A.N., Kim-Shapiro, D.B., Patel, R.P., Hogg, N., Shiva, S., Cannon, R.O. 3rd., Wink, D.A., Espey, M.G., Oldfield, E.H., Pluta, R.M., Freeman, B.A., Lancaster, J.R. Jr., Feelisch, M., and Lundberg, J.O.: The emerging biology of the nitrite anion. Nat. Chem. Biol., 1, 308-314, 2005.

[20] Caraceni, P., Ryu, H.S., Subbotin, V., De Maria, N., Colantoni, A., Roberts, L., Trevisani, F., Bernardi, M., and Van Thiel, D.H.: Rat hepatocytes isolated from alcohol-induced fatty liver have an increased sensitivity to anoxic injury. Hepatology, 25, 943-949, 1997. 